\title{
Desarrollo, planificación territorial y soportes materiales en Bolivia
}

\begin{abstract}
Jhohan Braxton Oporto Sánchez"
El siguiente texto caracteriza brevemente la relación entre los proyectos de desarrollo del Estado nación boliviano, la planificación territorial y el sistema de soportes materiales de la vida social en Cochabamba, particularmente a partir de tres procesos: 1) la Revolución Nacional, 1952-1984; 2) el régimen neoliberal, 1985-2005; y 3) el "proceso de cambio" impulsado por Evo Morales y su partido, el Movimiento Al Socialismo (MAS), de 2006 al presente.
\end{abstract}

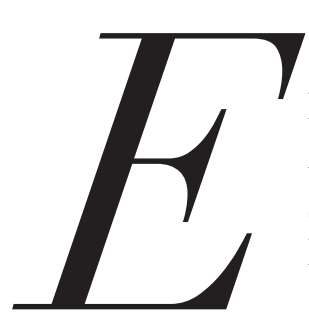

1 proceso de desarrollo socioeconómico en Bolivia puede analizarse mediante la relación $(\leftrightarrow)$ entre la planificación territorial (PT) y la planificación del sistema de soportes materiales de la vida social (SSM), donde " $\leftrightarrow$ " supone una interrelación complementaria, no una síntesis, en la que PT y SSM mantienen su identidad. El sistema de soportes materiales se refiere a:

*Estudiante del Doctorado de Estudios del Desarrollo de la Universidad Autónoma de Zacatecas. Correo electrónico: pipoqas@hotmail.com. aquellos objetos materiales, resultantes de un proceso voluntario y consciente de transformación de la naturaleza preexistente - de producción-, para satisfacer cualquiera de las necesidades sociales históricamente determinadas y estructuradas que se insertan inmóvil y durablemente sobre ella, dando lugar a la modificación de su estructura, su forma y funcionamiento, a la vez que sirven de condición particular, material, de la existencia y funcionamiento del elemento, proceso o relación social que determinó su producción y que se lo apropia. Su unidad y su carácter están dados y determinados por la relación entre objeto material y proceso de producción y apropiación por un elemento particular, diferenciado y diferenciable de la vida social. ${ }^{1}$ 
Mapa político de América Latina, con la ubicación de Bolivia y Cochabamba

Mapa de Cochabamba y sus zonas ecológicas
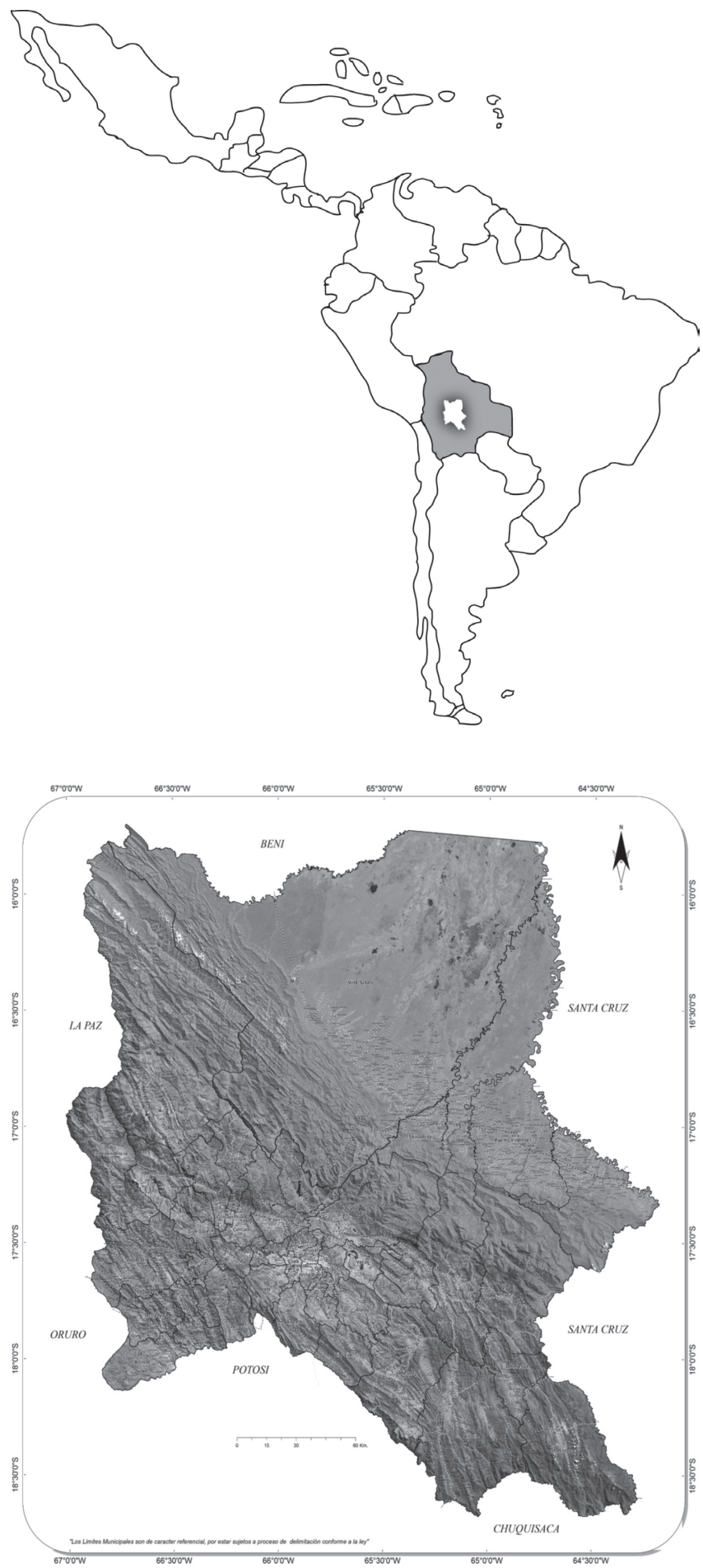

La dinámica reciente del desarrollo boliviano, particularmente en Cochabamba, pasa por tres proyectos 1) la Revolución Nacional de 1952 que, alineada con las políticas de desarrollo económico de posguerra, impulsa el patrón de acumulación de capital con interven- cionismo estatal; 2) con la crisis del modelo anterior, el Programa de Ajuste Estructural (PAE) y la aplicación de una nueva política económica (NPE) de corte neoliberal en 1985, alinean el patrón de acumulación hacia el libre mercado y la globalización; y 3) la crisis social, económica, política e ideológica del régimen neoliberal sienta las bases para el surgimiento de un proyecto de desarrollo alternativo al capitalista neoliberal: el "proceso de cambio" impulsado por el gobierno de Evo Morales, que desde $2006 \mathrm{al}$ presente se ha propuesto construir un camino de transición al socialismo comunitario sosteniéndose en la recuperación del Estado como agente central en la planificación del desarrollo, el control de recursos naturales, la industrialización y la redistribución equitativa de la riqueza.

\section{Periodo 1952-1984}

Imbuido en el proyecto de modernización, el Estado boliviano y los gobiernos posteriores a 1952 apostaron por políticas de industrialización y fortalecimiento del mercado interno. En tal sentido se promovieron iniciativas de Sssu y servicios para el establecimiento de fábricas en los centros urbanos de la región andina del país - La Paz, Oruro, Potosí y Cochabamba -, lo cual impulsó una alta emigración del campo a la ciudad. De manera complementaria, si bien la Reforma Agraria en 1953 supuso la titularización de tierra a favor de unidades campesinas, principalmente en el occidente del país por su densidad poblacional, la inexistencia de una política de planificación que apoye a la concreción de un ssu para el desarrollo agrícola y social en el campo dio continuidad a la situación de marginación que aqueja a la población.

Ante la previsible limitación de las políticas del Estado boliviano, el asesoramiento de Estados Unidos y su cooperación para del desarrollo definió que la ampliación de la frontera productiva no limitada a la explotación minera se debería extender al oriente del país con la consolidación de un eje transversal troncal - La Paz, Cochabamba y Santa Cruz - , y así articular la explotación de recursos naturales con el Océano Pacífico vía Chile. En esta lógica, el camino Cochabamba-Santa Cruz se desplegó como soporte material clave para la colonización de los llanos amazónicos del Chapare por el campesinado andino y hacia Santa Cruz la distribución de tierra permitió al capital extranjero y nacional adquirir grandes porciones sobre las que se desarrollará la agroindustria. 
Durante este periodo, la planificación, ejecución y seguimiento estuvieron a cargo del Estado y sus reparticiones departamentales por medio de corporaciones de desarrollo, y el presupuesto dependía del Tesoro General de la Nación y el financiamiento de la cooperación al desarrollo de instituciones como el Fondo Monetario Internacional (FMI), el Banco Mundial (BM) o el Banco Interamericano de Desarrollo (BID).

En el ámbito rural o campo, el ssu corresponde a la articulación e integración del territorio y de los centros productivos agrícolas mediante la apertura de vías, construcción de puentes, represas para producción de energía y riegos, electrificación y saneamiento básico. En el ámbito urbano o ciudad, la planificación del SSM se realizó de acuerdo a los principios de la modernidad urbanística europea, definiendo de esta manera una zonificación de industria, vivienda, comercio, recreación y servicios.

En síntesis, durante este periodo, al amparo del Estado, las políticas públicas se dirigieron hacia una PT $\leftrightarrow$ SSM más o menos coordinada, pero sin resolver las contradicciones entre el proyecto de modernización, la división estructural entre lo rural y lo urbano, las condiciones sociales y económicas internas y los intereses del capital nacional y extranjero.

\section{Mapa de la Organización \\ de los Estados Americanos (OEA) \\ del Proyecto Chapare - Propuesta \\ de Estudio para el Desarrollo Integrado (1975)}

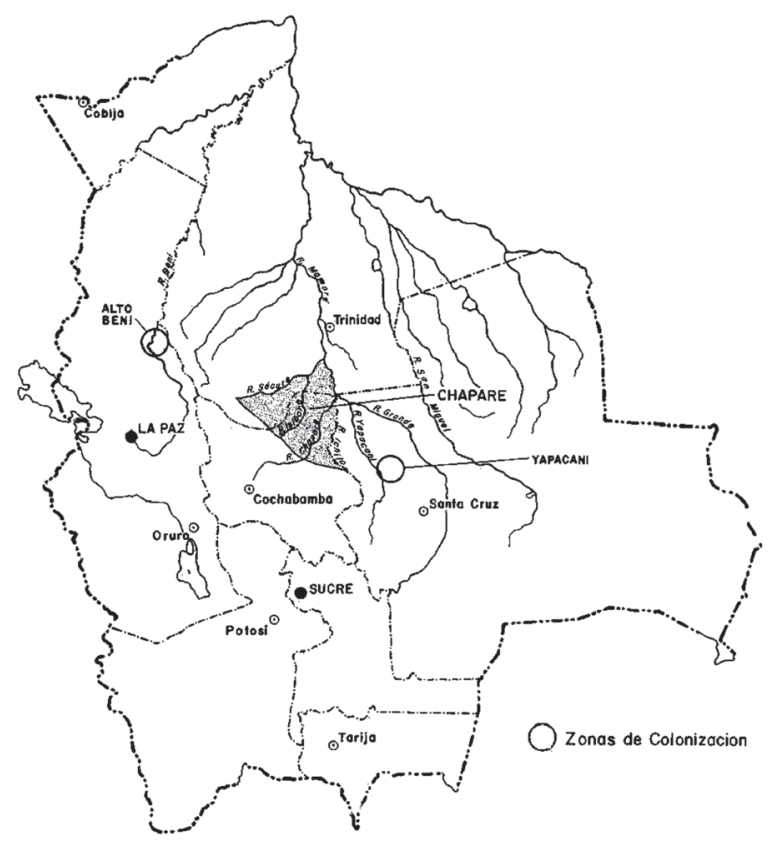

Fuente: OAS.org
Periodo 1985-2005

Por neoliberalismo se entiende:

una teoría de prácticas político-económicas que afirma que la mejor manera de promover el bienestar del ser humano, consiste en no restringir el libre desarrollo de las capacidades y de las libertades empresariales del individuo, dentro de un marco institucional caracterizado por derechos de propiedad privada, fuertes mercados libres y libertad de comercio. El papel del Estado es crear y preservar el marco institucional apropiado para el desarrollo de estas prácticas. ${ }^{2}$

\section{Red IIRSA Pacífico-Atlántico}

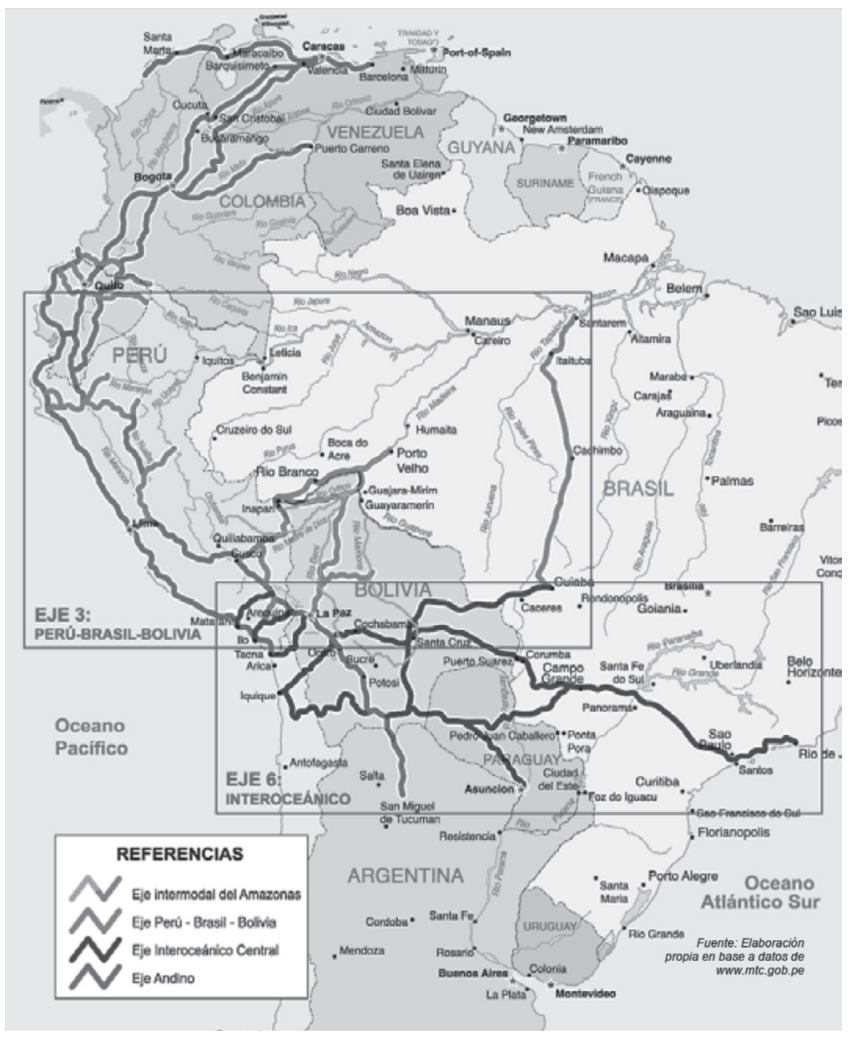

(Fuente: Villegas, 2013:91)

Las líneas generales del PAE neoliberal que se aplicaron en Bolivia en 1985 con la NPE sintetizada en el Decreto 21060, fueron: 1) definición de medidas de estabilización de corto plazo, austeridad fiscal y devaluación de la moneda destinadas a establecer la estabilidad macroeconómica (en términos de cuentas y precios) y promover las exportaciones; 2) liberalización de los mercados, del comercio y los flujos de capital entre y dentro de las naciones; 3) desregulación del sector privado, éste pasa a ser visto como el motor del sistema económico; 4) privatización de los medios 
de producción, reversión de la nacionalización de la industria que marcara el desarrollo en las décadas anteriores, y entrega de las empresas estatales al sector privado; 5) modernización del Estado, disminuyendo sus operaciones y reduciendo su papel económico a la prestación de apoyo al mercado, y el sector privado, su conductor y fuerza motriz. ${ }^{3}$

Tales medidas tendrán un efecto sustancial en la reconfiguración del territorio nacional, su planificación y la del ssu. Reducido el Estado a tareas de legislación de garantías de la propiedad privada y del libre flujo de capital en el mercado interno y su apertura al capital extranjero, la promoción de la "libre iniciativa" privilegiará en este periodo la anarquía del pragmatismo sobre la planificación. Dos efectos se desprenden de la caracterización de las líneas anteriores: 1) las privatizaciones, la flexibilización laboral y el ataque a las organizaciones sindicales provocará un gran número de despidos produciendo, inmediatamente, la aglomeración poblacional pauperizada por inmigración y búsqueda de fuentes de trabajo en el sector informal de las ciudades del eje troncal La Paz, Cochabamba y Santa Cruz; 2) en el nuevo marco normativo, frente al repliegue del Estado en tareas de PT y SsM son las "libres iniciativas" de actores privados empresariales y de organizaciones no gubernamentales (ONG) de la sociedad civil las que se convertirán en impulsores del pragmatismo de la rentabilidad capitalista y el beneficio particular en detrimento del bienestar colectivo.

Las inversiones en el SSM privilegian una visión no de articulación interna sino de articulación al mercado exterior, por ello son relevantes los proyectos y obras destinados a concretar un corredor bioceánico Pacífico-Atlántico consistente en una carretera y un tren para el transporte de carga pasando por el eje Santa Cruz, Cochabamba y La Paz. El proyecto responde a la iniciativa del BID y la Iniciativa de Integración Regional de Sur América (IIRSA).4 Sin embargo, la privatización de las empresas estatales de energía eléctrica y trenes, así como la fragmentación de la gestión pública mediante las leyes de participación popular (1994) y descentralización administrativa (1995), postergaron una vez más el proyecto, y en sentido contrario, producto de la desatención, se concretaron daños irreversibles, como la destrucción del tramo Cochabamba-Oruro por falta de inversiones y mantenimiento.

De manera interna, la descentralización administrativa, además de producir la fragmentación territorial, trajo la fragmentación de los presupuestos y, con ello, una articulación del Ssu asimétrico entre regiones con mayores y menores recursos por sus actividades productivas, población y dimensión de la jurisdicción territorial. Centros de salud, unidades educativas, sistemas de riego, electrificación, vías y transporte, entre otros SSM de las dinámicas socioeconómicas se sometieron a la capacidad de las organizaciones sociales y no-gubernamentales rurales o urbanas para negociar financiamiento estatal o de instituciones extranjeras.

En Cochabamba, un paradigma resultante de esta dinámica es la Guerra del Agua en 2000. Ante la privatización del agua, los regantes de las áreas rurales y los habitantes de las periurbanas se unieron para revertir el contrato entre el consorcio transnacional Bechtel y el Estado. Regantes y habitantes toleraron la desatención del Estado en la resolución de soportes adecuados a su demanda de agua para consumo en riego y hogares, financiando por cooperación extranjera sus proyectos; sin embargo, la privatización del recurso vital evidenció la perversa política económica del modelo neoliberal.

\section{Estado de las vías del ferrocarril Cochabamba-Oruro hacia 2002}
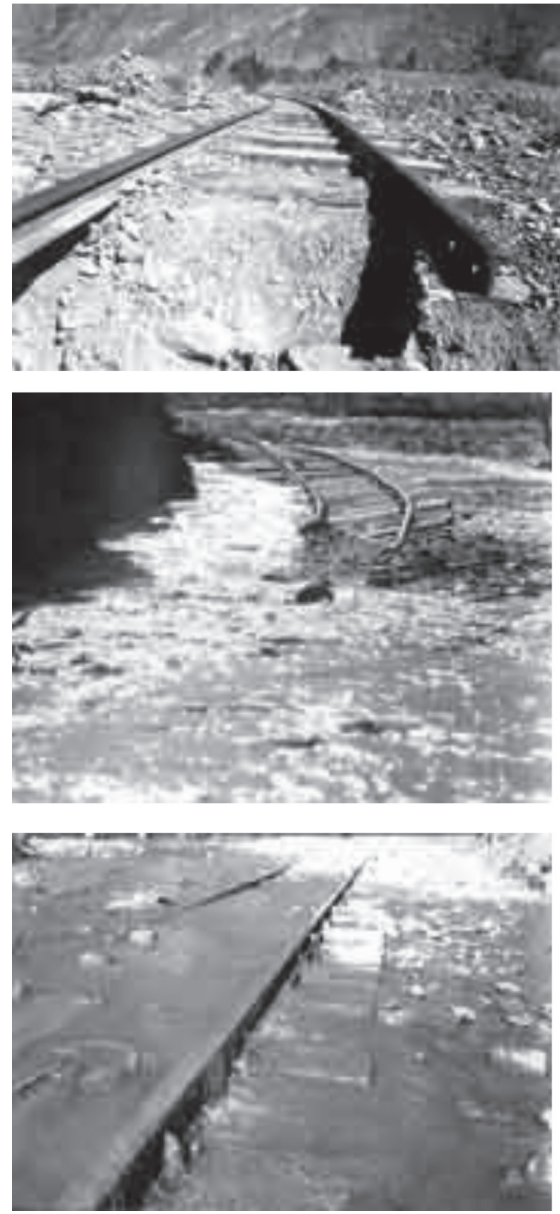

Fuente: Opinión 8/29/2002) 
Al influjo del libre mercado, las políticas públicas se dirigieron hacia una PT $\leftrightarrow$ SSM liberada a la conveniencia de los intereses particulares privados, por tanto limitada en su coordinación y promoviendo la continuidad y profundización de las contradicciones en el proyecto de modernización, la división estructural entre lo rural y lo urbano, la pauperización y su expresión material en sus soportes urbano-rurales.

\section{Periodo 2006 al presente}

La Revolución Democrática y Cultural encabezada por Evo Morales y el mas en 2006 emergió como un proyecto político alternativo con una crítica al desarrollo y acumulación capitalista y una mirada histórica más profunda al proceso colonial; por tanto, se reivindica como un proyecto anticolonial y antiimperialista. En el discurso y la práctica se ha propuesto la instrumentación de:

un modelo económico acorde a las características del país, tomando en cuenta a la diversidad de actores, su particular estructura social y económica, la asimetría regional, la exclusión política económica y social y los altos niveles de pobreza. Para ello, el Estado propicia la recuperación de los recursos naturales, promueve las actividades de los distintos actores sociales y económicos y redistribuye los ingresos con criterios de equidad lo cual está transformando sustancialmente el país. ${ }^{5}$

Existen muchos indicadores económicos (PIB, reservas, crecimiento del mercado interno) y sociales (alfabetismo, salud, asistencia social) que dan cuenta de que el "proceso de cambio" ha logrado avances sustanciales y que apuntalan el papel del Estado como agente central del desarrollo. Producto del incremento de rentas, principalmente por la nacionalización de recursos naturales (petróleo y gas), precios internacionales de materias primas, así como las alianzas estratégicas con países como Venezuela y organismos financieros internacionales como la Corporación Andina de Fomento (CAF), el Banco Interamericano de Desarrollo (BID), BM, una gran cantidad de fondos se han destinado a la puesta en marcha de proyectos de múltiples ámbitos del desarrollo económico y social.

En el caso de la PT $\leftrightarrow$ SSM, la dimensión material del proyecto desarrollo, se aprecia cierto pragmatismo que da cuenta de la persistencia de una vía no plani- ficada de desarrollo en semejanza al periodo neoliberal desplegado las dos décadas anteriores. Si bien el proyecto se ha sido respaldado con instrumentos de planificación que integran lo económico y social con lo territorial, la presión social y política se ha convertido en instrumento de decisión en el corto plazo sobre lo estratégico de mediano y largo plazos. Al presionar al Estado, las otrora fuerzas de cambio han promovido una emergente cultura de pragmatismo en la concreción de soportes materiales articulados al territorio sin una visión sistémica o integral. Numerosos son los ejemplos desplegados por todo el territorio nacional: complejos deportivos, unidades educativas básicas y superiores, sistemas viales, puentes, sistemas de riego, energía.

\section{Proyectos prioritario de inversión bases de la industrialización.}

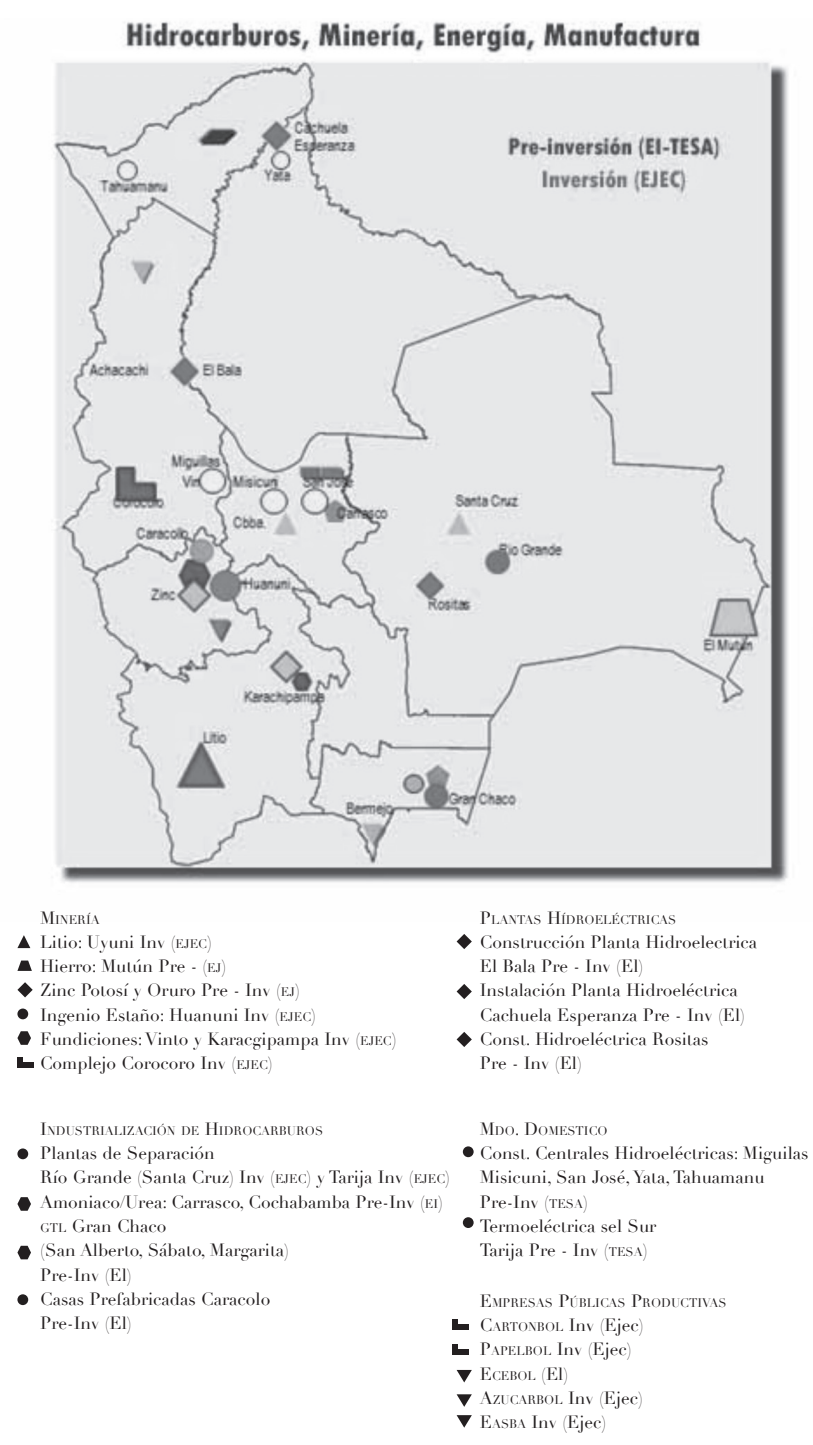

Fuente: Viceministerio de Inversión Pública y Financiamiento Externo (VIPFE), Bolivia. 


\section{Colegio modelo en municipio}

de Colomi (Abril de 2012)

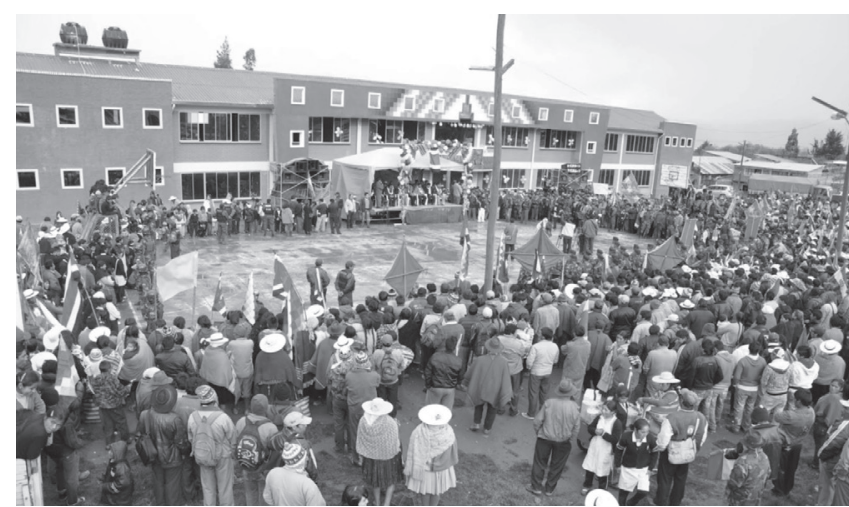

(Fuente: АвI 23/03/2012

En los inicios del gobierno de Morales se definió una PT $\leftrightarrow$ SSM a nivel nacional a partir de los recursos naturales estratégicos y su industrialización, por ejemplo, hierro en el Mutún en Santa Cruz, petroquímica en Carrasco en Cochabamba, litio en Uyuni Oruro, entre otros, para la generación de excedentes y empleo, produciendo así un efecto territorial distribuido e integral. Sin embargo, a ocho años de gobierno, a pesar de haber estabilizado la macroeconomía del país y alcanzado un crecimiento por encima de 5 puntos de 2011 al presente, los proyectos de petroquímica y litio recién iniciaron sus procesos, y casos como el del hierro se estancaron dando paso a microproyectos como los arriba mencionados.

En la dimensión territorial de Cochabamba, las políticas públicas de la PT $\leftrightarrow$ SSM se decantan por la vía pragmática del gobierno central. En consecuencia, proliferan microproyectos en oposición a emprendimientos mayores de integración intrarregional y nacional, y si acaso existen como aquellos vinculados a la revolución vial o la industrialización, se convierten en tareas truncas en el mediano plazo por ser producto de la efervescencia política y el discurso de reivindicación cultural originaria.

\section{Coliseo en la localidad de Totora}

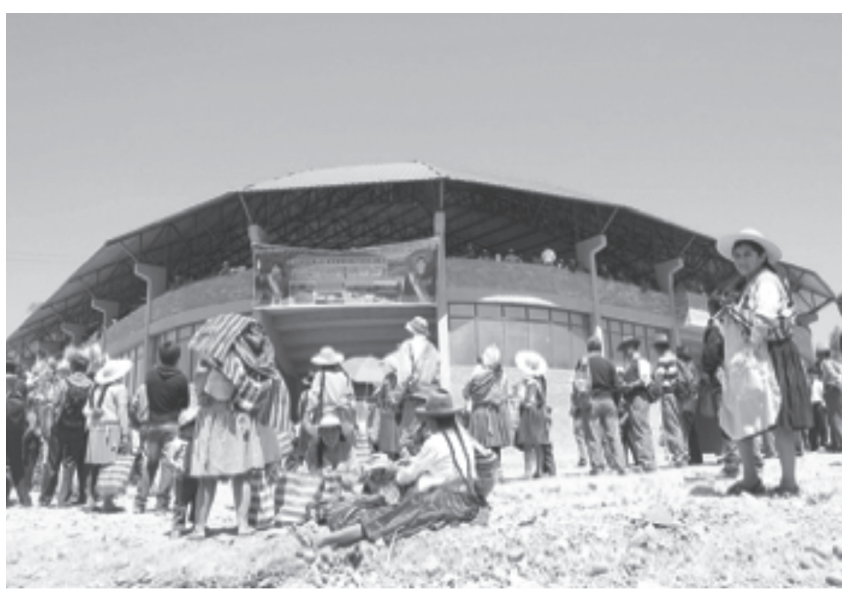

Fuente: Cambio 10/09/2012

En síntesis, se reconocen cambios sustanciales en el SSM que no acaban de articularse a una PT y de desarrollo de mediano y largo plazos. Por tanto, el proyecto de transformación de la economía, vía industrialización, no parece tener una base de condiciones generales de producción y reproducción social sobre la cual se pueda desplegar el desarrollo fuera del sistema capitalista propuesto en la retórica política del proyecto de Evo Morales y el mas. La transición hacia el sistema societal socialista comunitario o la construcción del Vivir Bien aparecen como una tarea cuyos contradictorios avances carecen de una base sólida que permita su pervivencia más allá de la actual coyuntura política. Más aún si se siguen las vías desplegadas por la faceta del capitalismo neoliberal.

\section{$-\operatorname{notas} \cdot-$}

'Emilio Pradilla (1984), Contribución a la crítica de la 'teoría urbana'. Del 'espacio' a la 'crisis urbana, México, UAM, p. 92.

${ }^{2}$ David Harvey ([2005]2007), Breve historia del neoliberalismo, Madrid, Akal, p. 6.

${ }^{3}$ Henry Veltmeyer y Juan Tellez (2001), "The State and Participatory Development in Bolivia", en Henry Veltmeyer y Anthony O'Malley (coords.), Trascending Neoliberalism, Kumarian Press, p. 68.
${ }^{` P}$ Pablo Villegas (2013), Geopolítica de las carreteras y el saqueo de los recursos naturales, Cochabamba, CEDIB.

${ }^{5}$ MAS-IPSP (2009), 2010-2015 Programa de gobierno. Bolivia avanza, p. 13, en http://issuu.com/boliviaavanza/docs/01._programa_evo_bolivia_avanza_2010-2015, Consultado el 25 de agosto de 2013. 